\title{
Evaluation of Therapeutic Efficacy of Ascorbic Acid in Patients with Bronchial Asthma
}

\author{
Razi Akhtar ${ }^{*}$, Sandeep Jain², Abhishek Kumar², Saurabh Bhatia', Jagdish C Joshi', Dhirendra Singh', Md Shamsuzzaman ${ }^{1}$, Meenakshi \\ Sharma ${ }^{1}$, Giridhari Pal', V K Vijayan ${ }^{3}$, S N Gaur ${ }^{3}$, Kavita Gulati ${ }^{4}$, Arunabha Ray ${ }^{4}$ \\ 'Department of Pharmacology, Vallabhbhai Patel Chest Institute (University of Delhi), Delhi 110007, INDIA. \\ 2Department of Pharmacology, University College of Medical Sciences (University of Delhi), Delhi 110095, INDIA. \\ ${ }^{3}$ Professor, Department of Pulmonary Medicine, Vallabhbhai Patel Chest Institute, (University of Delhi), Delhi 110007, INDIA. \\ ${ }^{4}$ Professor, Department of Pharmacology, Vallabhbhai Patel Chest Institute (University of Delhi), Delhi 110007, INDIA.
}

\begin{abstract}
Background: Asthma is a chronic inflammatory airway disease and oxidative stress may be involved in its pathogenesis. The present study aims at evaluating the therapeutic effects of antioxidant drug Ascorbic Acid (Vitamin C) in patients of Bronchial Asthma. Method: Patients of Bronchial asthma from outpatient facility of our institute were enrolled for the study and divided into study and control group. Both groups were given inhaled fluticasone and salmeterol and oral theophylline. Ascorbic acid was given in the study group. Each patient was followed up weekly for the assessment of efficacy parameters using pulmonary function test (PFT), clinical symptoms and emergency drug use. Oxidative stress parameters were assessed through the estimation of malondialdehyde levels and superoxide dismutase activity in the above two patient groups. Results: Although there was statistically significant improvement in pulmonary function tests $(p<0.001)$, clinical symptoms $(p<0.05)$ and emergency drug use $(p<0.001)$ in both treatment groups at the end of 4 weeks but there was no significant difference in these parameters between the two groups. There was
\end{abstract}

statistically significant improvement in malondialdehyde level $(p<0.001)$ and superoxide dismutase activity $(p<0.001)$ in ascorbic acid group only at the end of 2 weeks and 4 weeks. Conclusion: Ascorbic Acid failed to show any significant improvement in the patient symptoms and pulmonary function tests in the study group; however it showed statistically significant improvement in the status of Oxidative Stress.

Key words: Bronchial Asthma, Oxidative Stress, Antioxidants, Ascorbic Acid, Malondialdehyde, Superoxide Dismutase.

Correspondence :

Dr Razi Akhtar,

G-72 Muradi Road, Jamia Nagar, Delhi 110025, INDIA.

Phone no: +91-8447225208

E-mail: dr.razi.akhtar@gmail.com

DOI: 10.5530/jyp.2016.3.8

\section{INTRODUCTION}

Bronchial asthma is a major global health problem and affects approximately 300 million persons worldwide and more than 200,000 die of asthma annually. ${ }^{1}$ Oxidative stress is believed to play an important role in the pathogenesis of Bronchial Asthma. ${ }^{2}$ It's not clear whether the enhanced oxidative stress observed in asthma subjects is caused by inflammation or is a causative factor in the pathogenesis of the disease. However many recent reports have supported the critical role of oxidative stress in the development of various chronic immunologic diseases. ${ }^{3}$ In bronchial asthma, oxidative stress increase proinflammatory mediators causing airway inflammation, enhance bronchial hyperresponsiveness causing bronchospasm and it also increases mucin secretion..$^{4-8}$ Although several studies have emphasized the role of enhanced oxidative stress in the pathogenesis of airway inflammation, the therapeutic benefit of many antioxidants on airway inflammation has been found to be limited at best. ${ }^{4-8}$ These observations do not necessarily imply a negligible role of oxidative stress, rather they reflect the need to investigate proper antioxidants for airway inflammation and their therapeutic benefits for the underlying disease condition. It is necessary to clarify the precise role of oxidative stress in the development of asthma and identify means to use antioxidants as a treatment tool in bronchial asthma patients.

Ascorbic acid or vitamin $\mathrm{C}$ is an effective antioxidant. The ascorbate ion is predominantly responsible for antioxidant effects of ascorbic acid. It is a potential scavenger of free radicals such as superoxide radical and singlet oxygen. Free radical scavenging activity of suspected antioxidants is often compared with ascorbic acid as standard. ${ }^{9}$ It is also a coenzyme of other free radical scavenging enzymes like superoxide dismutase. This result in protection against lipid peroxidation of both cellular and intracellular membranes, thus making ascorbic acid an important component of overall antioxidant mechanism found in cells and tissues. ${ }^{4,10}$
We decided to investigate the beneficial effect of this time tested antioxidant in patients with bronchial asthma. The present study was designed to evaluate the anti oxidant effect of vitamin $\mathrm{C}$ and its therapeutic benefit in patients of Bronchial Asthma. Here we compare efficacy of two different regimens for treatment of bronchial asthma-a regimen of fluticasone, salmeterol and theophylline with a regimen of fluticasone, salmeterol, theophylline and ascorbic acid.

\section{MATERIALS AND METHODS}

The study was carried out jointly by the Department of Pharmacology and Vishwanathan Chest Hospital, Vallabhbhai Patel Chest Institute (VPCI). Data was collected between July 2011 and January 2012. The study was conducted as per the Good Clinical Practices (GCP) guidelines and the study protocol had the approval of the Institutional Ethical Committee. Written informed consent was obtained from the participating subjects prior to enrolling them for the study.

\section{Study population}

Patients visiting OPD of our institute who were diagnosed with bronchial asthma by the physician on the basis of previous history, clinical symptoms, spirometry and x-ray. In total, 86 patients of bronchial asthma from outpatient facility of the VPCI who satisfied inclusion criteria were enrolled for the study. Finally 50 patients were used for primary analysis (Figure 1).

\section{Patients' inclusion criteria}

1. Age group 18-60 years, irrespective of gender.

2. Patients with a clinical diagnosis of bronchial asthma.

3. Patients who have given written informed consent to participate in the study.

4. Patient relatively stable, ambulatory and cooperative. 


\section{Patients' exclusion criteria}

1. Patients below 18 years and above 60 years.

2. Pulmonary tuberculosis: past/present.

3. Pregnant/lactating females.

4. Patients with any systemic disease such as diabetes mellitus, rheumatic heart disease, renal disease, liver disease etc.

\section{Study Design}

This was a prospective, open label, parallel design study to monitor efficacy of Ascorbic Acid in outpatients of bronchial asthma undergoing standard treatment with inhaled corticosteroid Fluticasone 2-4 puffs 12 hourly, $44 \mathrm{mcg} /$ puff, inhaled long acting $\beta_{2}$ agonist (LABA) Salmeterol 1-2 puffs 12 hourly, $50 \mathrm{mcg} /$ puff, oral Theophylline $400 \mathrm{mg}$ sustained release once daily and inhaled short acting $\beta_{2}$ agonist (SABA) Levosalbutamol 2-4 puffs, $50 \mathrm{mcg} /$ puff SOS for exacerbation, along with tablet Ascorbic Acid $500 \mathrm{mg} 12$ hourly in study group only. Patients were enrolled after written informed consent as per inclusion and exclusion criteria. Medical history was taken and diagnosis was made on the first visit. Basal parameters like pulse, blood pressure (BP), respiratory rate, temperature, general physical examination were recorded and complete blood count (CBC) with hemoglobin ( $\mathrm{Hb}$ ), fasting blood sugar (FBS), liver function test (LFT), kidney function test (KFT), pulmonary function test (PFT) and X-ray were done. After enrollment, patients were divided into two groups and two different treatment regimens were given. The treatment groups were as under:

Group 1 (Control Group) $\rightarrow$ Inhaler (Fluticasone+Salmeterol)+Theop hylline

Group 2 (Study Group) $\rightarrow$ Inhaler (Fluticasone+Salmeterol)+Theophy lline+Ascorbic acid

Additionally inhaler levosalbutamol SOS was used as emergency medicine for acute exacerbation in both groups. Weekly follow-up was done during treatment for a period of four weeks. At each follow-up patients were enquired for new complaints and symptoms, and general physical examination was performed. Serum levels of malondialdehyde (MDA) and superoxide dismutase (SOD) as parameters of oxidative stress were measured at zero, 2 weeks and 4 weeks. PFT parameters (e.g.) forced expiratory volume in $1 \mathrm{sec}$ (FEV1), forced vital capacity (FVC) and FEV1/FVC ratio as parameter of treatment efficacy were measured at zero, 2 weeks and 4 weeks. Patients were enquired about the use of emergency medication, such as short-acting $\beta_{2}$ agonists to combat exacerbations, if any (Figure 2).

\section{Diagnosis and Monitoring Of Bronchial Asthma Patients}

Diagnosis of bronchial asthma is essentially a clinical exercise, supplemented by results of supportive investigations. A combination of information collected from clinical presentation, detailed medical history, careful physical examination and laboratory investigations was used to make the diagnosis of bronchial asthma in the current study. Measurement of lung function using spirometry and particularly the demonstration of reversibility of obstructive airflow pattern greatly enhance diagnostic confidence. Detailed monitoring of symptoms such as cough, dyspnea, sputum and acute exacerbation requiring inhaled or nebulised salbutamol was done at zero, 2 and 4 weeks.

\section{Measurement of Lung Function}

Measurement of forced expiratory volume in $1 \mathrm{sec}$ (FEV1), forced vital capacity (FVC) and FEV1/FVC ratio was done in our study at zero, 2 and 4 weeks of treatment. Predicted values of FEV1, FVC, and FEV1/ FVC based on age, sex, and height have been obtained from population studies. In current study the values of FEV1, FVC and FEV1/FVC were measured as percentage of normal predicted values based on age, sex, and height of the individual.

\section{Malondialdehyde (MDA)}

Malondialdehyde is a marker of lipid peroxidation. Lipid peroxidation is a well-established mechanism of cellular injury, and is used as an indicator of oxidative stress in tissues and body fluids. The MDA estimation was performed by the method as described by Okhawa et $a l^{11}$ and Protein estimation was performed by the method as described by Lowry et al. ${ }^{12}$ The data was expressed as $\mathrm{pmol} / \mathrm{mg}$ of serum proteins at zero, 2 and 4 weeks.

\section{Superoxide Dismutase (SOD)}

SOD is a free radical scavenging enzyme which scavenges peroxide radical, the most important free radical responsible for oxidative damage. SOD is a protective enzyme against oxidative stress and its levels are found to be low in several chronic diseases. In the present study the assay of SOD was performed by method as devised by Nandi and Chatterjee. ${ }^{13}$ SOD activity was calculated by the method of Marklund and Marklund. ${ }^{14}$ The activity was expressed as units/gm protein; 1 unit corresponds to the amount of enzyme needed to inhibit the auto oxidation of pyrogallol by $50 \%$.

\section{STATISTICAL ANALYSIS}

Data on continuous measurements is presented as mean \pm standard deviation (SD) and data on categorical measurements is presented as Number (\%). Significance is assessed at $5 \%$ level of significance $(P$-values $<0.05$ ). Student $t$ test (two tailed, independent) has been used to find the significance of study parameters on continuous scale between two groups. Leven 1s test for homogeneity of variance has been performed to assess the homogeneity of variance and Student $t$ test (two tailed, dependent) has been used to find the significance of study parameters on continuous scale within each group. Chi-square/Fisher Exact test has been used to find the significance of study parameters on categorical scale between two groups. Repeated Measures ANOVA has been used to find the significance of SOD and MDA within each group.

\section{RESULTS}

\section{Demographic study}

Both groups were comparable in demographic study. Analysis of the data showed that there was no significant difference in the age, gender distribution and severity of asthma in both groups (Table 1).

\section{Efficacy Study}

A comparative evaluation of all 3 spirometry parameters (i.e.) FEV1, FEV1/FVC and FVC in both groups showed that there was a noticeable improvement at the end of the trial (4 weeks), as compared with the baseline values $(\mathrm{p}<0.001)$. However at the end of 2 weeks and 4 weeks, the improvement in these parameters in both groups was similar and there was no significant statistical difference $(p>0.05)$. So addition of ascorbic acid to standard treatment failed to show statistically significant improvement in spirometry parameters when compared to standard treatment with inhaled steroid, inhaled long acting $\beta_{2}$-agonists, oral methylxanthine and inhaled short acting $\beta_{2}$-agonists SOS (Table 2).

On comparison of symptoms' score which comprised of severity of cough, dyspnea and quantity of sputum that was inquired from the patients at each follow-up visit and accordingly scored ranging from 1 (nil) to 4 (severe) for cough and dyspnea and 1 (no sputum production) to 4 ( $>45 \mathrm{ml}$ sputum production daily). At the end of study period total score was calculated for all the follow-up visits of each patient in both groups. It was seen that there was a significant improvement in symptoms of both groups $(\mathrm{p}<0.05)$ but there was no significant difference between the two groups ( $\mathrm{p}>0.05)$ (Table 2). 


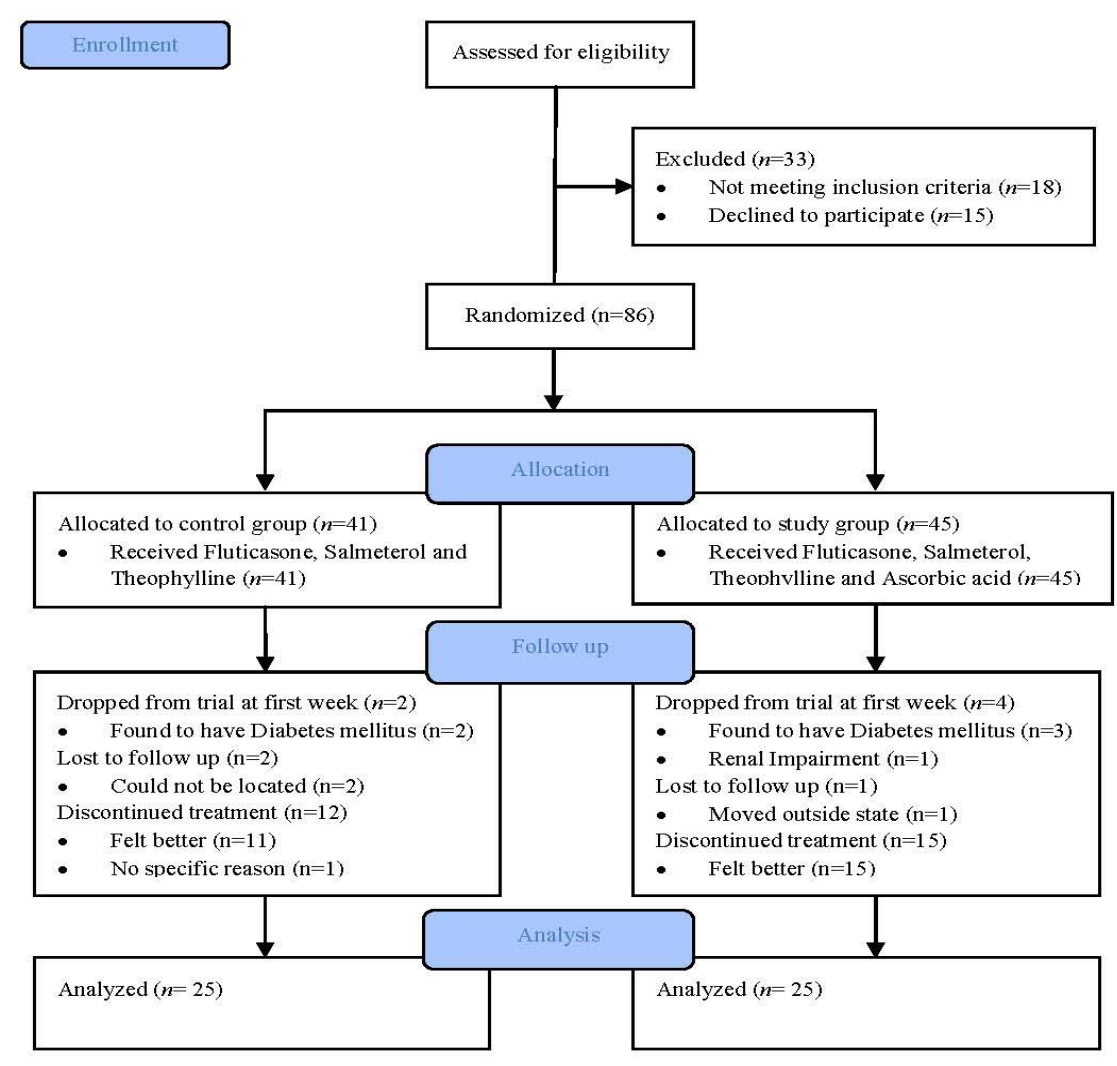

Figure 1: Study Cohort.

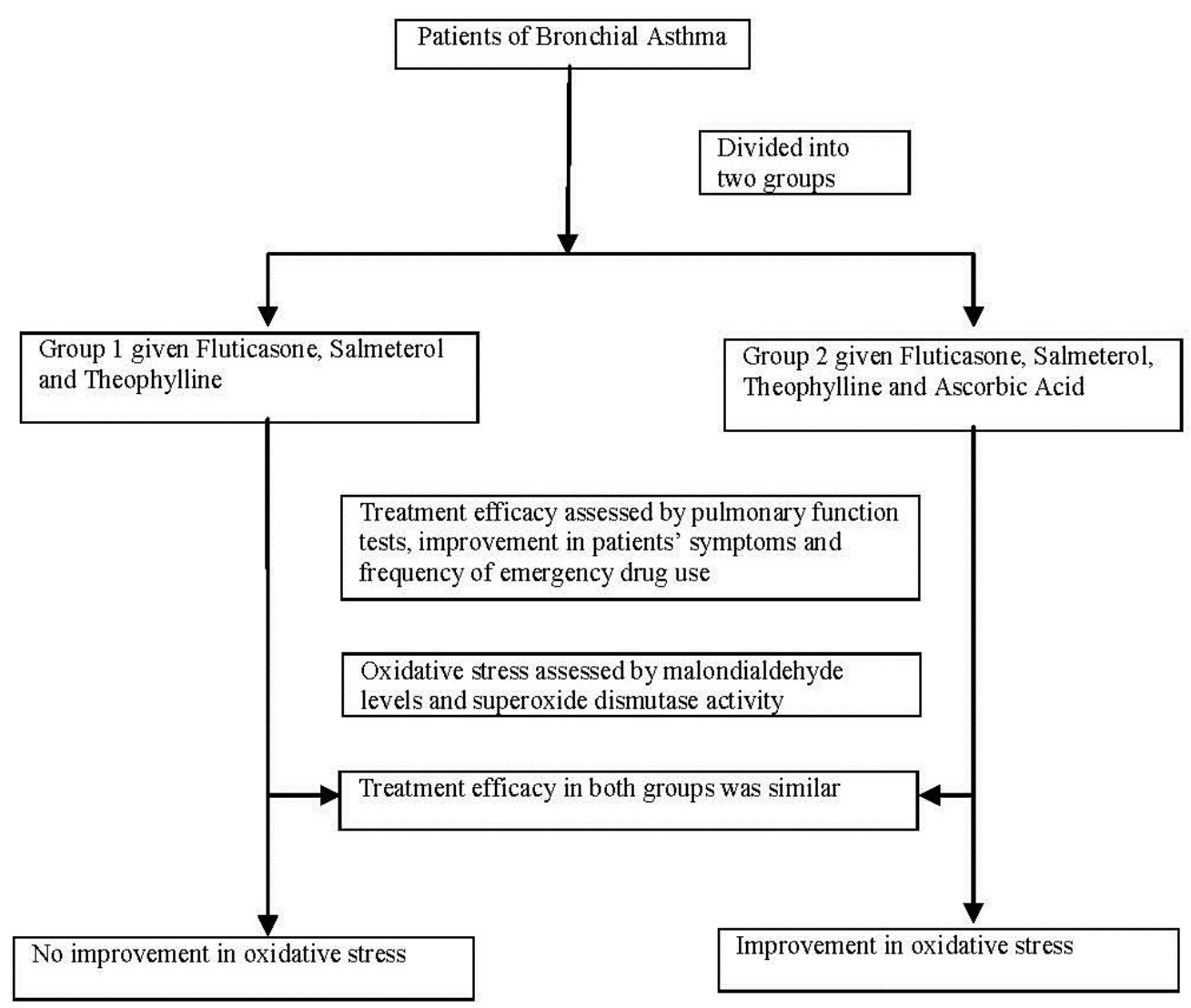

Figure 2: Diagrammatic Abstract. 
Table 1: Baseline characteristics of study population at enrollment

\begin{tabular}{|c|c|c|}
\hline Characteristic & $\begin{array}{l}\text { Fluticasone-salmeterol- } \\
\text { theophylline } \\
(\mathrm{N}=25)\end{array}$ & $\begin{array}{l}\text { Fluticasone-salmeterol- } \\
\text { theophylline-ascorbic acid } \\
\qquad(\mathrm{N}=25)\end{array}$ \\
\hline \multicolumn{3}{|l|}{ Age-yr } \\
\hline 19 to $20-$ no. (\%) & $4(16.0)$ & $2(8)$ \\
\hline 21 to $30-$ no. (\%) & $7(28.0)$ & $11(44.0)$ \\
\hline 31 to $40-$ no. $(\%)$ & $7(28.0)$ & $7(28.0)$ \\
\hline 41 to 50 -no. (\%) & $6(24.0)$ & $3(12.0)$ \\
\hline 51 to 60 -no. (\%) & $1(4.0)$ & $2(8.0)$ \\
\hline \multicolumn{3}{|l|}{ Gender } \\
\hline Male-no. (\%) & $11(44.0)$ & $11(44.0)$ \\
\hline Female-no. (\%) & $14(56.0)$ & $14(56.0)$ \\
\hline \multicolumn{3}{|l|}{ Asthma Severity } \\
\hline Mild-no. (\%) & $13(52.0)$ & $12(48.0)$ \\
\hline Moderate-no. (\%) & $7(28.0)$ & $7(28.0)$ \\
\hline Moderate-no. (\%) & $5(20.0)$ & $6(24.0)$ \\
\hline
\end{tabular}

Samples are age matched $(\mathrm{p}=0.918)$, gender matched $(\mathrm{p}=1.000)$ and severity of asthma matched $(\mathrm{p}=1.000)$.

\section{Table 2: Efficacy Outcomes}

\begin{tabular}{|c|c|c|c|}
\hline Outcome & $\begin{array}{l}\text { Fluticasone-salmeterol-theophylline. } \\
\qquad(\mathrm{N}=25)\end{array}$ & $\begin{array}{l}\text { Fluticasone-salmeterol- } \\
\text { theophylline-ascorbic acid. }(\mathrm{N}=25)\end{array}$ & $p$ value \\
\hline \multicolumn{4}{|c|}{ FEV1-\% of normal } \\
\hline Initial & $75.68 \pm 17.22$ & $74.12 \pm 15.35$ & 0.737 \\
\hline 2 weeks & $89.40 \pm 12.80$ & $88.40 \pm 13.26$ & 0.787 \\
\hline 4 weeks & $90.12 \pm 12.14$ & $89.36 \pm 12.83$ & 0.831 \\
\hline \multicolumn{4}{|c|}{ FEV1/FVC - \% of normal } \\
\hline Initial & $79.16 \pm 14.53$ & $76.72 \pm 13.09$ & 0.536 \\
\hline 2 weeks & $90.48 \pm 11.86$ & $90.20 \pm 10.26$ & 0.929 \\
\hline 4 weeks & $91.16 \pm 11.79$ & $90.88 \pm 10.47$ & 0.930 \\
\hline \multicolumn{4}{|c|}{ FVC-\% of normal } \\
\hline Initial & $81.08 \pm 11.38$ & $83.4 \pm 10.12$ & 0.450 \\
\hline 2 weeks & $97.96 \pm 5.32$ & $98.68 \pm 7.28$ & 0.692 \\
\hline 4 weeks & $99.08 \pm 4.96$ & $98.60 \pm 6.58$ & 0.772 \\
\hline \multicolumn{4}{|c|}{ Cough-no. of participants/total no. (\%) } \\
\hline Initial & $19 / 25(76)$ & $17 / 25(68)$ & 0.529 \\
\hline 2 weeks & $4 / 25(16)$ & $4 / 25(16)$ & 1.000 \\
\hline 4 weeks & $3 / 25(12)$ & $3 / 25(12)$ & 1.000 \\
\hline \multicolumn{4}{|c|}{ Sputum-no. of participants/total no. (\%) } \\
\hline Initial & $13 / 25(52)$ & $12 / 25(48)$ & 0.777 \\
\hline 2 weeks & $3 / 25(12)$ & $3 / 25(12)$ & 1.000 \\
\hline 4 weeks & $1 / 25(4)$ & $0 / 25(0)$ & 1.000 \\
\hline \multicolumn{4}{|c|}{ Dyspnea-no. of participants/total no. (\%) } \\
\hline Initial & $19 / 25(76)$ & $20 / 25(80)$ & 0.733 \\
\hline 2 weeks & $4 / 25(16)$ & $4 / 25(16)$ & 1.000 \\
\hline 4 weeks & $3 / 25(12)$ & $3 / 25(12)$ & 1.000 \\
\hline \multicolumn{4}{|c|}{$\begin{array}{c}\text { Emergency drug use-no. of participants/total } \\
\text { no. (\%) }\end{array}$} \\
\hline Initial & $14 / 25(56)$ & $13 / 25(52)$ & 1.000 \\
\hline 2 weeks & $8 / 25(32)$ & $7 / 25(28)$ & 1.000 \\
\hline 4 weeks & $7 / 25(28)$ & $6 / 25(24)$ & 1.000 \\
\hline
\end{tabular}

FEV1, FEV1/FVC and FVC values are expressed as \% of normal. Results are presented in Mean \pm SD, post-hoc values are by Bonferroni. Level of significance between two groups was calculated by Student $t$ test (two tailed, independent).

Cough, sputum, dyspnea and emergency drug use values are presented as number (\%) of patients. Level of significance between two groups was calculated using Fisher Exact Test. 
Table 3: Parameters of Oxidative Stress

\begin{tabular}{cccc}
\hline Parameter & $\begin{array}{c}\text { Fluticasone-salmeterol- } \\
\text { theophylline. }(\mathrm{N}=25)\end{array}$ & $\begin{array}{c}\text { Fluticasone-salmeterol- } \\
\text { theophylline-ascorbic } \\
\text { acid. }(\mathrm{N}=25)\end{array}$ & p value \\
\hline $\begin{array}{c}\text { MDA Serum Conc. } \\
\text { (pmol/mg protein) }\end{array}$ & $47.17 \pm 9.78$ & & \\
Initial & $51.46 \pm 10.18$ & $46.06 \pm 9.72$ & 0.688 \\
2 weeks & $51.38 \pm 10.29$ & $36.86 \pm 5.80$ & $<0.001$ \\
4 weeks & $14.65 \pm 3.07$ & $36.08 \pm 5.14$ & $<0.001$ \\
SOD Serum Conc. & & & \\
(U/gm protein) & $14.25 \pm 2.61$ & $14.46 \pm 3.01$ & 0.904 \\
Initial & $14.40 \pm 2.77$ & $19.17 \pm 3.01$ & $<0.001$ \\
2 weeks & & $19.77 \pm 2.84$ & $<0.001$ \\
\hline 4 weeks & & & \\
\hline
\end{tabular}

Malondialdehyde (MDA) and Superoxide dismutase (SOD) results are presented in Mean \pm SD, Post-hoc values are by Bonferroni.

Level of significance between two groups was calculated by Student $t$ test (two tailed, independent).

The incidence of acute exacerbations and use of emergency medications was significantly decreased in both groups as compared to baseline $(\mathrm{p}<0.001)$. However the use of emergency medications was similar in both groups (Table 2).

\section{Parameters of Oxidative Stress}

MDA levels were significantly decreased in group 2 as compared to group 1 at both 2 weeks and 4 weeks time. There was also significant decrease in MDA levels in study group at 2 weeks and 4 weeks as compared to initial values (Table 3 ).

SOD levels were significantly increased in group 2 as compared to group 1 at both 2 weeks and 4 weeks time. The increase in serum SOD levels in group 2 at 2 weeks and 4 weeks as compared to initial values was also found to be statistically significant (Table 3 ).

\section{DISCUSSION}

In the present study efforts were made to investigate the therapeutic effect of ascorbic acid in patients of bronchial asthma. Ascorbic acid clearly demonstrated improvement in parameters of oxidative stress as evident by the decrease in MDA and increase in SOD levels. However this improvement in oxidative stress by ascorbic acid didn't show any therapeutic benefit. Pulmonary function tests and clinical symptoms in ascorbic acid group failed to show any significant improvement as compared to control group. As ascorbic acid has no established bronchodilatory or anti inflammatory effect; in view of the present study its use in patients of bronchial asthma appears fruitless and will only result in increased cost of treatment without any beneficial effect.

\section{CONCLUSION}

The findings of the present study indicate no therapeutic effect of ascorbic acid in bronchial asthma.

\section{LIMITATION}

We could not compare the efficacy of treatment with ascorbic acid alone with efficacy of treatment with inhaled fluticasone and salmeterol as ethics do not allow us to with hold standard treatment with inhaled steroid and long acting $\beta 2$ agonist in patients with bronchial asthma.

\section{ACKNOWLEDGEMENTS}

We acknowledge the timely help of all Residents of Department of Pulmonary Medicine, Vallabhbhai Patel Chest Institue, who helped in diagnosis and follow up of patients during study period. We also extend sincere gratitude towards Prof K K Pillai, Dr Razi Ahmad and Dr Deepti Chopra, Department of Pharmacology, Hamdard Institute of Medical Sciences \& Research, Jamia Hamdard, for helping with publication process.

\section{CONFLICT OF INTEREST}

There is no conflict of interest between the authors.

Funding: No special funding required for the study.

Importance of the study: The current study supports the existing knowledge that although oxidative stress is associated with several chronic diseases but its improvement with antioxidants does not improve the disease outcome.

\section{ABBREVIATIONS USED}

MDA: Malondialdehyde; SOD: Superoxide dismutase; GCP: Good clinical practices; LABA: Long acting beta agonist; SABA: Short acting beta agonist; PFT: Pulmonary function test; FEV1: Forced expiratory volume in 1 second; FVC: Forced vital capacity. 


\section{REFERENCES}

1. Ait-Khaled N, Enarson D, Bousquet J. Chronic respiratory diseases in developing countries: the burden and strategies for prevention and management. Bul World Health Organ. 2001;79(10):971-9.

2. Cho Y, Moon H. The Role of Oxidative Stress in the Pathogenesis of Asthma. Allergy Asthma Immunol Res. 2010;2(3):183.

3. Stone J, Yang S. Hydrogen Peroxide: A Signaling Messenger. Antioxid Redox Signal. 2006;8(3-4):243-70.

4. Omenaas E, Fluge Ã , Buist A, Vollmer W, Gulsvik A. Dietary vitamin C intake is inversely related to cough and wheeze in young smokers. Respir Med. 2003;97(2):134-42

5. Grievink L, Smit H, Ocke M, van `t Veer P, Kromhout D. Dietary intake of antioxidant (pro)-vitamins, respiratory symptoms and pulmonary function: the MORGEN study. Thorax. 1998;53(3):166-71.

6. Brown D, Griendling K. Nox proteins in signal transduction. Free Radic Biol Med. 2009;47(9):1239-53

7. Terada L. Specificity in reactive oxidant signaling: think globally, act locally. J Cell Biol. 2006;174(5):615-23.
8. Li N, Nel A. Role of the Nrf 2-Mediated Signaling Pathway as a Negative Regulator of Inflammation: Implications for the Impact of Particulate Pollutants on Asthma. Antioxid Redox Signal. 2006;8(1-2):88-98.

9. Agarwal K, Varma R. Antioxidant Ability of Some Common Indian Vegetables. J Young Pharm. 2015;7(3):262-6.

10. Bendich A, Machlin L, Scandurra O, Burton G, Wayner D. The antioxidant role of vitamin C. Adv Free Radio Biol Med. 1986;2(2):419-44.

11. Ohkawa H, Ohishi N, Yagi K. Assay for lipid peroxides in animal tissues by thiobarbituric acid reaction. Anal Biochem. 1979:95(2):351-8.

12. Lowry O, Rosebrough N, Farr A, Randall R. Protein measurement with the folin phenol reagent. J Biol Chem. 1951;193(1):265.

13. Nandi $A$, Chatterjee I. Assay of superoxide dismutase activity in animal tissues. J Biosci. 1988;13(3):305-15.

14. Marklund S, Marklund G. Involvement of the Superoxide Anion Radical in the Autoxidation of Pyrogallol and a Convenient Assay for Superoxide Dismutase. Eur J Biochem. 1974;47(3):469-74 